\title{
Antioxidant properties of rice bran oil from different varieties extracted by solvent extraction methods
}

\begin{abstract}
Antioxidant properties of rice bran oil from different rice bran varieties; Rice Bran-Bario (RB-Bario), Rice bran-Lowland (RB-Low) and Rice Bran- Upland rice (RB-Up), collected from different cultural plots, were assessed. Measurement of antioxidant properties was evaluated byTPC(total phenolic content), DPPH scavenging activities and reducing power of extracts.. The study shows that antioxidant efficacy of rice bran was found the highest in RBUp, followed by RB- Low and RB-Bario. The antioxidant properties were related to the rice bran origin and water irrigation demand by particular variety. $\mathrm{RB}-\mathrm{Up}$ has a unique plantation condition which takes least amount of water retention which contribute to the highest antioxidant activity. Extraction solvents used shows that Upland (16.15\%) and Lowland (16.16\%) yielded the highest amount in conserving the crude fat oil in rice bran extract compared to Bario.
\end{abstract}

Keyword: Antioxidant; Extraction; Rice bran oil 